\title{
Practical considerations for the operative team in performing tracheostomy during the COVID-19 pandemic
}

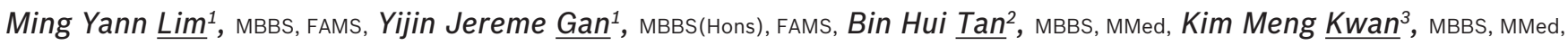
Quan Qing Richmond $\underline{\text { Lim }}^{1}$, MBBS, MMed, Sow Fong Lee $\underline{\text { Le }}^{4}$ MASc, Yaw Khian $\underline{\text { Chong }^{1}}$, MBBS, FAMS
\end{abstract}

\section{INTRODUCTION}

The ongoing COVID-19 pandemic has left hospitals around the world scrambling to design new workflows to improve safety for the healthcare worker. As up to $20.3 \%$ of COVID-19 patients require intensive care, ${ }^{(1)}$ the otolaryngologist will be called upon to perform tracheostomies for patients who require prolonged ventilatory support.

What are some of the practical measures that can be taken to reduce risk to operating theatre (OT) teams? Herein, we describe the workflow at Tan Tock Seng Hospital/National Centre for Infectious Diseases (TTSH/NCID) for an open tracheostomy for COVID-19-positive patients who require prolonged ventilatory support in the intensive care unit (ICU). This workflow reflects a combination of experience-based and evidence-based considerations.

The debate on whether percutaneous tracheostomy is less aerosol-generating is ongoing, and there is currently no consensus on whether it may represent an advantage over open tracheostomy. ${ }^{(2)}$ Percutaneous tracheostomy may be a good alternative to open tracheostomy with the right expertise and a patient with favourable anatomy. ${ }^{(3)}$ However, it is not the preferred technique at our institution, reflecting reservations on the part of our intensivists. The main reservations are: (a) potentially longer contact of airway with the external environment using the serial dilation technique; (b) longer duration of loss of positive end-expiratory pressure (PEEP) during the procedure in a PEEP-dependent patient; (c) potential dissemination of aerosols during the employment of bronchoscopy to aid percutaneous tracheostomy; and (d) increased risk to all should a complication, such as uncontrollable bleeding, occur from percutaneous tracheostomy.

\section{PREOPERATIVE MEASURES}

Before the tracheostomy, it is advisable to ensure that the endotracheal tube (ETT) aspirates or nasopharyngeal swabs have been tested for severe acute respiratory syndrome coronavirus 2 (SARS-CoV-2) and are repeatedly negative. At our institution, we require a minimum of two consecutive polymerase chain reaction (PCR)-negative aspirates 24 hours apart. The median time for viral PCR to become negative is 11 days, but this can be up to one month in severe disease. ${ }^{(4)}$ However, the median time from symptom onset to severe hypoxaemia and ICU admission is approximately 7-12 days. ${ }^{(5)}$ Hence, most patients should become PCR-negative by three weeks from intubation. Given the paucity of evidence for performing early tracheostomy and balancing the risk to medical personnel, ${ }^{\left({ }^{6}\right)}$ we recommend waiting at least three weeks from intubation to achieve swab negative status before performing tracheostomy. Although live virus has not been detected after Day 8 of illness onset in a small study, ${ }^{(7)}$ such a recommendation reflects a cautious approach to maximise safety to healthcare providers. This recommendation is also consistent with the American Academy of Otolaryngology guideline on tracheostomies during the COVID-19 pandemic. $^{(8)}$

Since the time of the SARS pandemic in 2003, our institution has improved on our pandemic preparedness in terms of infrastructure and workflow. We have divided our patients who need tracheostomies into two groups: a high-risk group with COVID-19-positive status on ETT aspirate and a lower-risk group whose COVID-19 status is negative at the time of surgery. For patients who remain COVID-19 positive, two special OTs have been built in an adjacent connected building, the NCID. These theatres are designed to be negative pressure environments with a higher frequency of air exchange compared to a standard OT, with exiting air passing through a high-efficiency particulate air (HEPA) filter. In COVID-19 patients who remain repeatedly and consistently swab negative on ETT aspirate or swab, the tracheostomy can technically be performed in a standard major OT, although preferably one with a higher frequency of air exchanges and where the exiting air continues to be filtered by a HEPA filter into the external environment. In our previous experience with SARS, tracheostomy was performed by the ICU bedside. ${ }^{(9)}$ During this pandemic, we have opted to perform it in the OT. Although it is technically feasible for the surgery to be performed in the ICU, it is not ideal, as space is limited, with suboptimal positioning of the operating team with respect to the patient.

Apart from changes in the OT setup, all the precautions subsequently described apply both to swab negative and swab positive patients. This reflects our caution in declaring a patient COVID-19 negative, as there have been a few reported cases of subsequent positive PCR tests who had been declared negative in PCR tests performed 24 hours apart. ${ }^{(10)}$ Before scrubbing, the surgeon dons a powered air-purifying respirator (PAPR). This is done in a pressure neutral corridor outside the scrub room.

${ }^{1}$ Department of Otolaryngology, Head and Neck Surgery, Tan Tock Seng Hospital, ${ }^{2}$ Department of Anaesthesia, Woodlands Health Campus, ${ }^{3}$ Department of Anaesthesiology, Intensive Care and Pain Medicine, ${ }^{4}$ Healthcare Management, Tan Tock Seng Hospital, Singapore

Correspondence: Dr Lim Ming Yann, Senior Consultant, Department of Otolaryngology, Head and Neck Surgery, Tan Tock Seng Hospital, 11 Jalan Tan Tock Seng, Singapore 308433. ming_yann_lim@ttsh.com.sg 
Although there has been no definite evidence to show that PAPR reduces viral transmission compared to the N95 mask, in theory, the use of HEPA filters within a PAPR offers a higher level of respiratory protection. ${ }^{(11)}$ Consistent with this, PAPR is employed at our institution when a tracheostomy is performed for a COVID-19 patient. It is important to understand that the optimum use of PAPR requires appropriate preparatory training.

During patient transfer, specific and clearly demarcated routes must be designated. If existing design dictates a shared corridor, special protocols must be instituted to ensure that there is no 'random collision' of unsuspecting and unprotected staff or other patients. Prior to patient transfer from the ICU to the OT, the anaesthetist administers an anaesthetic and a neuromuscular blocking agent to prevent spontaneous ventilation, coughing or gagging of the patient during transfer. This allows patientventilator synchrony and prevents aerosolisation of potentially infectious secretions. When the breathing circuit is disconnected from the ICU ventilator to be connected to the transport ventilator and later from the transport ventilator to the OT ventilator, the endotracheal tube is clamped with a pair of forceps to ensure minimal aerosolisation to the environment. The tracheostomy should be performed by an experienced surgeon to reduce operating time and potential morbidity requiring unnecessary return to the OT. In our department, we have appointed two surgeons (a consultant and a registrar) to perform tracheostomies for the first batch of patients. Depending on the volume of patients, we anticipate the need to appoint new paired teams every three to six months to avoid psychological fatigue among our surgeons.

In anticipation of the need for tracheostomies to be performed in COVID-19 patients, our surgical and anaesthetic teams have performed trial simulations. In these trial runs, the surgeons donned PAPR and performed routine tracheostomies in nonCOVID-19 patients. Donning a PAPR suit precludes wearing of a headlight, restricts movements, and affects the ability to hear verbal commands and alarms. Performing trial simulations enabled our surgeons to gain invaluable experience operating with the PAPR. Prior to our first tracheostomy in a COVID-19positive patient (ETT aspirates negative at time of surgery), an OT team huddle was conducted. Various important time points during the procedure were discussed with the anaesthetist, which allowed team members to know what to expect during the procedure.

\section{INTRAOPERATIVE MEASURES}

For the procedure proper, our usual practice is to alert the anaesthetist at least two minutes prior to entry into the trachea to allow adequate preoxygenation. Preoxygenation is especially important for a patient requiring a high level of ventilatory support, such as a patient who needs a high fraction of inspired oxygen or PEEP.

After preoxygenation, the trachea is only entered after (a) ventilation is stopped and the adjustable pressure-limiting valve is opened to release the PEEP, and (b) complete exhalation has been assessed to have taken place based on a zero-flow reading in the spirometry graph on the anaesthetic machine. Ventilation is only recommenced after placement of the tracheostomy tube, inflation of the cuff and reconnection of the breathing circuit. The use of a cuffed non-fenestrated tube is ideal for this instance. A cuff pressure of $25-30 \mathrm{cmH}_{2} \mathrm{O}$ should be maintained, and the surgeon should check with the anaesthetist if there is any leak in the breathing circuit.

\section{POSTOPERATIVE MEASURES}

After surgery, in both high- and low-risk scenarios, the surgeon unscrubs and removes the shoe cover, gloves and gown in a corner of the OT, preferably at least $2 \mathrm{~m}$ from the patient. Our OT for low-risk patients has an air circulation cycle of 31.8 times per hour; based on this, the risk of aerosol contamination would be sufficiently minimised to safely de-gown two minutes after the tracheostomy tube enters the trachea and the breathing circuit is re-established. De-gowning, by institutional protocol, is preferably performed in the OT, as the gown has been directly 'contaminated' through contact wisth the patient.

A specific step-by-step protocol for unscrubbing and doffing of PAPR based on infection control policy should be followed as per institutional guidelines. The PAPR and N95 mask should be removed outside of the main OT where the patient is located. At the NCID OT, for high-risk cases, there is an anteroom behind the major OT where the PAPR can be doffed by a trained assistant and the surgeon can take a shower thereafter in an inbuilt shower area. Current institutional protocols do not dictate that the operative team must go into isolation after the procedure due to the robustness of the personal protective equipment and safety measures employed.

When ventilating the patient postoperatively, it is always important to keep the cuff inflated adequately. The tracheostomy should not be disconnected from the breathing circuit unnecessarily. The use of a metered dose inhaler is preferable to nebulisation ${ }^{(12)}$ and an in-line suction catheter is preferable to open suctioning in managing secretions to reduce the risk of aerosolisation. Current guidelines suggest that the use of humidified wet circuits should be avoided to reduce the risk of contaminating the room if there is an unexpected circuit disconnection. ${ }^{(13)}$ In our institution, a heat and moisture exchanger with HEPA filter capabilities is used at the interface between the tracheostomy and the breathing circuit to keep the ventilated air humidified. If a tracheostomy change is required, patients should first be proven swab negative. When tracheostomy patients have been fully weaned off the ventilator, another heat and moisture exchanger filter may be placed over the tracheostomy. This is a highly efficient bacterial-viral filter that reduces viral shedding to the external environment. A summary of the measures to be considered in performing tracheostomy in a COVID-19-positive patient is shown in Table I.

\section{CONCLUSION}

It is important to acknowledge that the above algorithm applies to a centre that has adequate resources to follow these protocols. The COVID-19 pandemic is a global problem, with varied resources 
Table I. Precautionary measures for tracheostomy during the COVID-19 pandemic.

\begin{tabular}{|lll|}
\hline Pre- and perioperative & Intraoperative & Postoperative \\
\hline $\begin{array}{l}\text { Two negative endotracheal tube or } \\
\text { nasopharyngeal swabs }\end{array}$ & Negative pressure OT if available & $\begin{array}{l}\text { Appropriate de-gowning and doffing } \\
\text { of PPE }\end{array}$ \\
\hline $\begin{array}{l}\text { Administer anaesthetic and neuromuscular } \\
\text { blocking agent prior to transfer }\end{array}$ & Preoxygenate patient before trachea cut & $\begin{array}{l}\text { Keep tracheostomy cuff inflated at all } \\
\text { times during ventilation in ICU }\end{array}$ \\
\hline $\begin{array}{l}\text { Appropriate PPE for OT staff: PAPR (Versaflo) } \\
\text { or PAPR (Jupiter) + N95 mask }\end{array}$ & $\begin{array}{l}\text { Complete cessation of ventilation prior to } \\
\text { entering trachea }\end{array}$ & $\begin{array}{l}\text { Always keep circuit connected, use of } \\
\text { in-line suction catheter for suctioning }\end{array}$ \\
\hline Limit number of personnel in OT & $\begin{array}{l}\text { Cuff tracheostomy tube immediately post } \\
\text { insertion, ensure no air leak }\end{array}$ & $\begin{array}{l}\text { Use HME when fully weaned off } \\
\text { ventilator }\end{array}$ \\
\hline
\end{tabular}

HME: heat and moisture exchanger; ICU: intensive care unit; OT: operating theatre; PAPR: powered air-purifying respirator; PPE: personal protective equipment

available in different centres. Many centres may not have a specially designed negative pressure OT or some of the personal protective equipment described above. In such situations, it is best to defer tracheostomy, if possible, until the aspirates are swab negative, recognising a balance between ensuring the safety of healthcare workers and the benefits of early tracheostomy. Regardless, the abiding principles of reduction in aerosolisation risk and personal protection should be adhered to, as far as possible and depending on the resources available, to achieve maximum safety for the operating team.

\section{REFERENCES}

1. Rodriguez-Morales AJ, Cardona-Ospina JA, Gutiérrez-Ocampo E, et al. Clinical, laboratory and imaging features of COVID-19: a systematic review and metaanalysis. Travel Med Infect Dis 2020; 34:101623.

2. Jacob T, Walker A, Mantelakis A, Gibbins N, Keane O. A framework for open tracheostomy in COVID-19 patients. Clin Otolaryngol 2020; 45:649-51.

3. Givi B, Schiff BA, Chinn SB, et al. Safety recommendations for evaluation and surgery of the head and neck during the COVID-19 pandemic. JAMA Otolaryngol Head Neck Surg 2020; 146:579-84.

4. Chen J, Qi T, Liu L, et al. Clinical progression of patients with COVID-19 in
Shanghai, China. J Infect 2020; 80:e1-e6.

5. Phua J, Weng L, Ling L, et al. Intensive care management of coronavirus disease 2019 (COVID-19): challenges and recommendations. Lancet Respir Med 2020; 8:506-17.

6. Andriolo BN, Andriolo RB, Saconato H, Atallah ÁN, Valente O. Early versus late tracheostomy for critically ill patients. Cochrane Database Syst Rev 2015; 1:CD007271.

7. Wölfel R, Corman VM, Guggemos W, et al. Virological assessment of hospitalized patients with COVID-2019. Nature 2020; 581:465-9.

8. American Academy of Otolaryngology-Head and Neck Surgery. Tracheotomy Recommendations During the COVID-19 Pandemic. Available at: https://www. entnet.org/content/tracheotomy-recommendations-during-covid-19-pandemic. Accessed June 15, 2020.

9. Chee VW, Khoo ML, Lee SF, Lai YC, Chin NM. Infection control measures for operative procedures in severe acute respiratory syndrome-related patients. Anesthesiology 2004; 100:1394-8.

10. Sethuraman N, Jeremiah SS, Ryo A. Interpreting diagnostic tests for SARSCoV-2. JAMA 2020; 323:2249-51.

11. Roberts V. To PAPR or not to PAPR? Can J Respir Ther 2014; 50:87-90.

12. Respiratory Care Committee of Chinese Thoracic Society. [Expert consensus on preventing nosocomial transmission during respiratory care for critically ill patients infected by 2019 novel coronavirus pneumonia]. Zhonghua Jie He He Hu Xi Za Zhi 2020; 43:288-96. Chinese.

13. ENT UK. Guidance for surgical tracheostomy and tracheostomy tube change during the COVID-19 pandemic. Available at: https://www.entuk.org/ tracheostomy-guidance-during-covid-19-pandemic. Accessed June 15, 2020. 